\title{
THE WEB OF QUADRICS
}

BY T. R. HOLLCROFT

1. Introduction. A triply infinite linear system of quadrics was first defined by De Jonquières* in 1862. Such a linear system later became known as a web.

The characteristics of a web of quadrics, with and without basis elements, have never been completely or accurately given, although many mathematicians have investigated this problem during the past seventy-five years. $\dagger$ Also, the web with a basis double point, that is, the web of quadric cones, has not been treated.

The purpose of this paper is to give a brief summary of results, correcting errors and completing the sets of characteristics; also, to derive the characteristics of a web of quadric cones. References to more complete discussions of certain parts are given.

In all cases, the quadrics will be considered as loci of points. Each web type has its dual, whose treatment in plane coordinates is algebraically identical with that in point coordinates.

2. The Web and the Associated Involution. The web of quadrics has the equation

$$
\sum \lambda_{i} f_{i}=0, \quad(i=1,2,3,4),
$$

in which the $f_{i}=0$ are equations of quadrics with given basis elements. The web has a jacobian quartic surface $J$.

The quadrics of the web in a 3 -space $(x)$ are in $(1,1)$ correspondence with the planes of a 3 -space $(y)$. The correspondence is defined by the equations

$$
\rho y_{i}=f_{i}, \quad(i=1,2,3,4) .
$$

* De Jonquières, Journal de Mathématiques, (2), vol. 7 (1862), p. 412.

$\dagger$ Encyklopädie der Mathematischen Wissenschaften, vol. III $_{2}$, pp. 250254. Pascal, Repertorium der höheren Mathematik, vol. $\mathrm{II}_{2}$ (1922), pp. 629-631. Virgil Snyder and F. R. Sharpe, Space involutions defined by a web of quadrics, Transactions of this Society, vol. 19 (1918), pp. 275-290. 
This correspondence gives rise to an involution between the points of $(y)$ and $(x)$. The order of this involution is the number of non-basic intersections of three quadrics of the web. In the involution, the branch-point surface $L$ in $(y)$ is in $(1,1)$ correspondence with $J$, which is the coincidence surface in $(x)$. This involution is fully discussed in the paper by Snyder and Sharpe cited above.

The complete image of $J$ is $L$. The complete image of $L$ is $J$ counted twice and a residual surface $R$. The properties of the web are defined by the characteristics of the three surfaces $L$, $J$, and $R$.

The web contains $\infty^{3}$ nets and $\infty^{4}$ pencils of quadrics. The systems are in $(1,1)$ correspondence with the points (vertices of bundles of planes) and lines (axes of pencils of planes) of $(y)$, respectively. The jacobian curves of the nets of quadrics form a web of curves on $J$, whose images on $L$ are the contour curves of the associated tangent cones to $L$. Two jacobian curves of any two nets of the web have intersections whose number is equal to the order of the discriminant of a quadric of the web.

The characteristics of $L, J$, and $R$ are obtained by the methods of a former paper.* It is to be noted that, since the quadric web contains composite surfaces and this does not occur for $n>2$, the quadric web is a special web whose characteristics do not all result on substituting $n=2$ in the formulas for a general $n$.

For a web of quadrics, $L$ has neither bitangent planes nor stationary tangent planes and therefore neither a bitangential nor a parabolic curve.

The characteristics of $L$ that do not vanish are represented by the following symbols: $N$, order; $a$, order of tangent cone; $a^{\prime}$, class of plane section; $n^{\prime}$, class; $b$, order of nodal curve; $c$, order of cuspidal curve; $\rho$, class of nodal curve; $\sigma$, class of cuspidal curve; $\gamma$, number of intersections of $b$ and $c$ which are cusps on $b ; \uparrow \beta$, number of intersections of $b$ and $c$ which are cusps on $c$; $q$, rank of $b ; r$, rank of $c ; p_{1}$, genus of plane section; $\delta^{\prime}$, bitangents of plane section; $\kappa^{\prime}$, stationary tangents of plane section; $\delta$, nodal generators of tangent cone; $\kappa$, cuspidal generators of tan-

* T. R. Hollcroft, The general web of algebraic surfaces of order $n$ and the involution defined by it, Transactions of this Society, vol. 35 (1933), pp. 855-868.

$\dagger$ The same symbol will be used both as the order and name of a curve. 
gent cone; $C$, number of conic nodes; $C^{\prime}$, number of conic tropes.

The image of $b$ is the intersection curve $b_{1}$ (counted twice) of $J$ and $R$ and the nodal curve $b_{2}$ of $R$. The image of $c$ is the contact curve $c_{1}$ (counted three times) of $J$ and $R$ and a residual curve $c_{2}$ which is the cuspidal curve of $R$.

3. The Web with Simple Basis Points. The web of quadrics may have any number up to six of independent simple basis points. For any number of basis points, zero to six inclusive, the characteristics of $L$ are given in the following table.

\begin{tabular}{|c|c|c|c|c|c|c|c|}
\hline No. basis & 0 & 1 & 2 & 3 & 4 & 5 & 6 \\
\hline$N$ & 16 & 14 & 12 & 10 & 8 & 6 & 4 \\
\hline$n^{\prime}$ & 4 & 4 & 4 & 4 & 4 & 4 & 4 \\
\hline$a=a^{\prime}$ & 12 & 12 & 12 & 12 & 12 & 12 & 12 \\
\hline$p_{1}$ & 9 & 8 & 7 & 6 & 5 & 4 & 3 \\
\hline$\delta^{\prime}$ & 22 & 23 & 24 & 25 & 26 & 27 & 28 \\
\hline$\kappa^{\prime}$ & 24 & 24 & 24 & 24 & 24 & 24 & 24 \\
\hline$b$ & 60 & 40 & 24 & 12 & 4 & 0 & 0 \\
\hline$c$ & 36 & 30 & 24 & 18 & 12 & 6 & 0 \\
\hline$\gamma$ & 120 & 72 & 36 & 12 & 0 & 0 & 0 \\
\hline$\beta$ & 80 & 56 & 36 & 20 & 8 & 0 & 0 \\
\hline$t$ & 80 & 32 & 8 & 0 & 0 & 0 & 0 \\
\hline$q$ & 40 & 28 & 18 & 10 & 4 & 0 & 0 \\
\hline$r$ & 68 & 62 & 54 & 44 & 32 & 18 & 0 \\
\hline$\rho$ & 80 & 56 & 36 & 20 & 8 & 0 & 0 \\
\hline$\sigma$ & 32 & 32 & 30 & 26 & 20 & 12 & 0 \\
\hline$\delta$ & 28 & 28 & 28 & 28 & 28 & 28 & 28 \\
\hline$\kappa$ & 24 & 24 & 24 & 24 & 24 & 24 & 24 \\
\hline$C$ & 0 & 0 & 1 & 3 & 6 & 10 & 16 \\
\hline$C^{\prime}$ & 10 & 11 & 12 & 13 & 14 & 15 & 16 \\
\hline
\end{tabular}

The corresponding characteristics of $J$ and $R$ are given in the following table. In this table, "mult. $P_{i}$ on $J$ " means "the multiplicity of each simple basis point on $J$," and similarly for $R$ and the curves. 
When the web has six basis points, $J$ is a Weddle quartic surface and $L$ is a Kummer quartic surface.*

\begin{tabular}{|c|c|c|c|c|c|c|c|}
\hline$\underbrace{\begin{array}{c}\text { No. basis } \\
\text { points }\end{array}}_{J, R, \text { in }(x) .}$ & 0 & 1 & 2 & 3 & 4 & 5 & 6 \\
\hline order of $J$ & 4 & 4 & 4 & 4 & 4 & 4 & 4 \\
\hline mult. $P_{i}$ on $J$ & & 2 & 2 & 2 & 2 & 2 & 2 \\
\hline no. lines, $J$ & 10 & 10 & 11 & 13 & 16 & 20 & 25 \\
\hline order of $R$ & 24 & 20 & 16 & 12 & 8 & 4 & 0 \\
\hline mult. $P_{i}$ on $R$ & & 10 & 8 & 6 & 4 & 2 & 0 \\
\hline order of $c_{1}$ & 18 & 18 & 17 & 15 & 12 & 8 & 0 \\
\hline mult. $P_{i}$ on $c_{1}$ & & 6 & 5 & 4 & 3 & 2 & 0 \\
\hline order of $b_{1}$ & 60 & 44 & 30 & 18 & 8 & 0 & 0 \\
\hline mult. $P_{i}$ on $b_{1}$ & & 8 & 6 & 4 & 2 & 0 & 0 \\
\hline order of $c_{2}$ & 90 & 66 & 45 & 27 & 12 & 0 & 0 \\
\hline mult. $P_{i}$ on $c_{2}$ & & 12 & 9 & 6 & 3 & 0 & 0 \\
\hline order of $b_{2}$ & 120 & 72 & 36 & 12 & 0 & 0 & 0 \\
\hline mult. $\overline{P_{i}}$ on $b_{2}$ & & 24 & 12 & 4 & 0 & 0 & 0 \\
\hline
\end{tabular}

The image of each basis point is a plane of $(y)$ whose image in $(x)$ is the basis point and the quadric tangent cone to $J$ at the basis point. All lines joining basis points are lines of $J$.

4. The Web with Basis Curves. The web of quadrics may have the following basis curves or combinations of simple basis points and basis curves:

(1) Web with basis line $l$. The surface $J$ is a ruled quartic surface with the line $l$ three-fold. Every line of $J$ is the axis of a composite quadric. The only possible cones of the web are those whose vertices lie on $l$. The surface $L$ is the developable of the curve $c$, and has the characteristics: $N=6, a=a^{\prime}=4, n^{\prime}=0$, $b=4, c=6, \kappa^{\prime}=0, \delta^{\prime}=3, \kappa=0, \delta=0, \sigma=4, \rho=8, \beta=4, \gamma=0, t=0$, $r=6, q=6$. $R$ is a ruled quartic that does not contain $l$. The curve $c_{1}$ is of order 5 and intersects $l$ in four points. The curve $b_{1}$ is of order 6 and intersects $l$ in the same four points, which are the

* For a derivation of the equations of $J$ and $L$ and further particulars of this case, see Hudson, Kummer's Quartic Surface, 1905, pp. 169-172. 
intersections of $l$ and $R$. The cuspidal curve of $R$ is of order 3 . $R$ has no nodal curve. The image of $l$ is a quadric of $(y)$ whose generators correspond to the points of $l$.

(2) Web with two basis skew lines $l_{1}$ and $l_{2}$. This web contains no cones and any two planes through $l_{1}$ and $l_{2}$, respectively, constitute a composite quadric of the web. $J$ is, therefore, indeterminate. The lines $l_{1}$ and $l_{2}$ belong to the same regulus for each proper quadric. Three quadrics of the web have no intersections outside of the two basis lines and thus no involution is established.

(3) Two coplanar lines $l_{1}$ and $l_{2}$, intersecting at $P$. For all quadrics of the web, $l_{1}$ and $l_{2}$ belong to different reguli and the plane of these two lines is a common tangent plane at $P$.

The only cones of the web have their vertices at $P$ and contain $l_{1}$ and $l_{2}$ as generators. The axes of composite quadrics generate a quadric cone with vertex at $P$ and containing $l_{1}$ and $l_{2}$. This quadric cone and the plane of the basis lines counted twice constitute the surface $J$. The involution is of order two and $L$ is a quadric surface.

(4) A basis conic $c$. $J$ consists of the plane $\pi$ of $c$ counted twice and a quadric surface through $c . L$ is a quadric. The involution is of order two. The web contains no composite quadrics except $\pi^{2}$. Each cone of the web has its vertex and two generators on the quadric which is the proper $J$.

(5) One line and one or two basis points. If the web has one basis line $l$ and one basis point $P, J$ consists of a ruled cubic surface through $P$ with $l$ double and the plane determined by $l$ and $P$. The proper $J$ is the cubic surface. $L$ is a ruled quartic surface with a cuspidal cubic and no nodal curve. The involution is of order three. $R$ is a quadric cone with vertex at $P$; and $J$ and $R$ have a contact cubic $c_{1}$ through $P$.

If the web has one basis line $l$ and two basis points $P_{1}$ and $P_{2}$, $J$ consists of a quadric through $l$ and both basis points and two planes, both through $l$ and one each through $P_{1}$ and $P_{2}$. The involution is of order two. $L$ is a quadric.

If there are additional basis elements, such as seven points, one line and three points, two coplanar lines or a conic and one point, the web is homaloidal and the associated involution is birational. The characteristics of such webs are well known. 
5. Properties of the Web. The following discussion applies to all the preceding cases.

$J$ is the locus of vertices of cones, of axes of composite quadrics, and of all contacts of quadrics of the web.

The contact curve $c_{1}$ of $J$ and $R$ is the locus both of threepoint coincidences of the involution and of stationary contacts of pencils of quadrics of the web. The curves $c$ and $c_{1}$ are in $(1,1)$ correspondence.

The intersection curve $b_{1}$ of $J$ and $R$ is the locus both of pairs of coincidences of the involution and of pairs of simple contacts of pencils of quadrics of the web. The curves $b$ and $b_{1}$ are in $(1,2)$ correspondence.

The points $\gamma$ correspond to pairs of points $P_{1} P_{2}$ on $b_{1}$, through one of which, $P_{1}$, passes $c_{1}$. In the involution, to a point $\gamma$ corresponds a three-point coincidence at $P_{1}$ and a simple coincidence at $P_{2}$. In the web, there are $\gamma$ pencils of quadrics in which the quadrics have stationary contact at a point $P_{1}$ and simple contact at the associated point $P_{\mathbf{2}}$.

The points $\beta$ correspond to points of osculation of $b_{1}$ and $c_{1}$. Such a point is a four-point coincidence in the involution. In the web, there are $\beta$ pencils of quadrics in which the quadrics have tacnodal contact at a point of osculation of $b_{1}$ and $c_{1}$.

The points $t$ correspond to $t$ triplets of nodes on $b_{1}$. Each triplet is a set of three simple coincidences in the involution. In the web, there are $t$ pencils of quadrics such that in each pencil the quadrics have three simple contacts at the three points corresponding to a triple point of $b$.

In case the surface $L$ does not have certain singularities, the quadrics of the associated web do not have the contacts to which these singularities correspond. Further details are given in the paper cited in $\$ 2$.

6. The Web with a Double Basis Point. The web of quadrics with a double basis point $Q$ is a web of quadric cones with a common vertex, at $Q$. This web has different characteristics from those discussed above.

This web of quadric cones contains $\infty^{3}$ nets of quadric cones. Each net has a jacobian cubic cone $J_{i}$ whose vertex is at $Q$ and whose plane section is an elliptic cubic. The $\infty^{3} J_{i}$ form a web. Two $J_{i}$ intersect in nine generators of which three are the axes 
of the three pairs of planes of the pencil of quadric cones common to the two nets. The other six generators are common to all the $\infty^{3} \quad J_{i}$ and are thus basis lines of the web $J_{i}$.

The jacobian of a net of jacobian cones $J_{i}$ is a cone of order 6 , consisting of a quadric cone of the web and four planes through $Q$ and through the six basis lines of the jacobian web. The four planes and six lines through $Q$ form a complete four-plane.

The $(1,1)$ correspondence between the web of cones and the planes of $(y)$ does not determine an involution between the points of the two spaces. There is, however, a surface $L$ in $(y)$ whose image is the bundle of lines on $Q$. The bundle of lines on $Q$ may be considered the jacobian of the web of cones since any line through $Q$ may be the axis of a composite cone of the web. To a plane section of $L$ corresponds a quadric cone of the web.

The surface $L$ in $(y)$ is a Steiner's quartic. It has the characteristics: $N=4, n^{\prime}=3, a=a^{\prime}=6, b=3, c=0, \delta^{\prime}=4, \kappa^{\prime}=6, \kappa=9$, $\delta=0, t=1, C^{\prime}=4, j$ (pinch points of $\left.b\right)=6$.

The nodal curve $b$ consists of three nodal lines through one point, which is the triple point of $b$ and of $L$. There are two pinch points on each nodal line.

The images of the six pinch points are the six basis lines of the web of jacobian cones. There are six pencils of quadric cones of the web in each of which four generators of every quadric cone of the pencil are consecutive with one basis line.

The images of the four contact conics of the four conic tropes of $L$ are, respectively, the four planes through the six basis lines and $Q$. These four planes are the loci of the limiting positions of axes of composite cones whose planes coincide.

The image of $b$ is a cubic cone through the six basic lines of the jacobian web and with three nodal generators corresponding to the triple point on $b$. This cubic cone consists of three planes through $Q$ and is the diagonal trihedral of the basic complete four-plane. These three planes constitute the locus of pairs of lines through $Q$ such that the quadric cones of a pencil have contact along the lines of a pair. In the web, there is one and only one pencil of quadric cones in which the cones have contact along three lines. These lines are the edges of the diagonal trihedral.

The web of quadric cones with one basic generator $l$ gives 
rise to a web of jacobian cubic cones with $l$ as a common nodal generator and two other basis lines. The jacobian of a net of jacobian cones consists of a quadric cone of the web and two planes, each counted twice, both through $l$ and each through one of the two basis lines of the jacobian web. In $(y), L$ is a ruled cubic with the characteristics: $N=3, n^{\prime}=3, a=a^{\prime}=4$, $b=1, c=0, \kappa^{\prime}=3, \delta^{\prime}=0, \kappa=3, \delta=0, t=0, j=2, C^{\prime}=2$. The image of $b$ is a plane through the two basis lines, but not through $l$. The images of the two pinch points of $b$ are the two basis lines. The images of the two conic tropes are the two planes determined by $l$ and the two basis lines paired with $l$.

The web of quadric cones with two basis generators $l_{1}$ and $l_{2}$ gives a web of jacobian cubic cones, each consisting of the plane $l_{1} l_{2}$ and a quadric cone of the web. The jacobian of a net of these composite cubic cones consists of a quadric cone of the web and the plane $l_{1} l_{2}$ counted four times. In $(y)$, the surface $L$ is a quadric. The web has contacts only in the plane $l_{1} l_{2}$.

The web of quadric cones can have no more than two basis lines or two basis points. One or two basis points determine one or two basis generators, respectively, and thus the discussion of basis points reduces to that of the two preceding cases.

Wells College

\section{ERRATUM}

On page 692 of the October issue of this Bulletin, in lines 5 and 19 , change $k$ to $K$. 